\title{
Preventive maintenance models with random maintenance quality
}

\author{
Shaomin $\mathrm{Wu}^{1}$, Derek Clements-Croome \\ School of Construction Management and Engineering, The University of Reading, \\ RG6 6AW, Reading, The United Kingdom
}

\begin{abstract}
In real-world environments it is usually difficult to specify the quality of a preventive maintenance $(\mathrm{PM})$ action precisely. This uncertainty makes it problematic to optimise maintenance policy. This problem is tackled in this paper by assuming that the quality of a PM action is a random variable following a probability distribution. Two frequently studied models are, a failure rate PM model and an age reduction PM model, and then will be investigated. The optimal PM policies are presented and optimised. Numerical examples are also given.
\end{abstract}

Key words: Preventive maintenance; long-run average cost; failure rate; maintenance quality

\section{Introduction}

Maintenance actions can generally be divided into two types: corrective maintenance $(\mathrm{CM})$ and preventive maintenance $(\mathrm{PM})$. The quality of maintenance $\overline{1}$ Corresponding author. Current E-mail: s.m.wu@kent.ac.uk 
actions in both $\mathrm{CM}$ and $\mathrm{PM}$ is an interesting research topic in the reliability literature, and is also vitally important when maintenance policies are being developed in practice.

The state of a piece of equipment after a maintenance action is performed is assumed to be one of the three situations: perfect, imperfect, and minimal. A perfect maintenance action is assumed to restore the equipment to be as good as new; an imperfect maintenance action may bring the equipment to any condition between as good as new and as bad as previously, and a minimal maintenance action is assumed to restore the equipment to a state the same as before the action. Examples of models for perfect, imperfect and minimal maintenance actions are Renewal Processes, Generalized Renewal Processes and Non-Homogeneous Poisson Processes, respectively. More comprehensive discussion in maintenance from both theoretical and application points of view can be found in Pham and Wang[1]; Dekker[2] and Scarf[3].

The assumption that the equipment can be restored imperfectly, or imperfect maintenance, is closer to many practical scenarios than the other two assumptions. For modelling the quality of a PM action, two approaches have often been studied: a failure rate PM model by Lie and Chun [4] and Nakagawa [5], and an age reduction PM model by Canfield [6] and Malik [7]. Based on these two models, Lin et. al. $[13,14]$ introduced a hybrid PM model that combines the failure rate PM model and the age reduction PM model.

Assume that PM actions on the equipment are carried out at every time interval $T$ independent of the failure history of the equipment, and CM actions are conducted upon failures. The failure rate PM model, the age reduction PM model and the hybrid PM model are defined as follows. 
- Failure Rate PM Model [4,5]. The failure rate after the $k$ th PM becomes $h_{k}(t)=\theta h_{k-1}(t)$ for $t \in(0, T)$, where $\theta(>1)$ is the adjustment factor, $h_{k}(t)$ $(t \in(0, T))$ is the failure rate after the $k$ th $\mathrm{PM}$, and $T$ is the time interval between two adjacent PM actions. Each PM resets the failure rate to zero and the rate of increase of the failure rate gets higher after each additional PM. This model considers the change of the slope of the failure rate function. In this model, the adjustment factor $\theta$ is an index for measuring the quality of PM.

- Age Reduction PM Model [6,7]. Canfield[6] and Malik[7] introduced age reduction models. In the age reduction model introduced by Canfield[6], the effective age after the $k$ th PM reduces to $t_{k}-\eta$ if the equipment's effective age was $t_{k}$ just prior to this $\mathrm{PM}$, where $\eta\left(<t_{k}\right)$ is the restoration interval in the effective age of the equipment due to the $k$ th PM. The restoration interval $\eta$ in this model is an index for measuring the quality of PM. This model has been widely used to optimize cost for maintenance policies under various assumptions[8,9]. In the age reduction model introduced by Malik[7], the effective age after the $k$ th PM reduces to $b t_{k}$ if the equipment's effective age was $t_{k}$ just prior to this PM, where $b<1$. Applications of the Malik model on the maintenance effect and optimizing maintenance policy can be found in $[11,12]$.

- Hybrid PM Model [13]. The failure rate after the $k$ th PM becomes $a_{k} h\left(b t_{k}+\right.$ $x)$, where $t_{k}$ is the time when the $k$ th $\mathrm{PM}$ is conducted, $1=a_{0} \leq a_{1} \leq a_{2} \leq$ $\ldots \leq a_{N-1}, 0=b_{0} \geq b_{1} \geq b_{2} \geq \ldots \geq b_{N-1}<1, x>0$, and $h(t)$ is the failure rate of the equipment when there is no CM or PM. Here, parameter $a_{k}$ plays the same role as the parameter $\theta$ in the failure rate PM model, and parameter $b_{k}$ functions similarly as the parameter $b$ in the Malik's age reduction PM model. 
All of the above three models assume that the failure rate of the equipment is increasing with time when no PM is conducted. This paper only studies the failure rate PM model and the Canfield age reduction PM model.

The parameters that determine the PM quality are the adjustment factor $\theta$ in the failure rate PM model and the restoration interval $\eta$ in the Canfield age reduction PM model. They are important because they impact on the frequency of PM's and therefore the long-run average cost. These parameters can be estimated based on a domain expert's suggestion [7] or real data [6]. It is assumed by prior research on the above two models that the parameters $\theta$ and $\eta$ are fixed constant. This assumption may be violated in many scenarios, especially in the case when the parameters are estimated by domain experts. It can be more practical to assume that these two parameters are random variables following certain probability distributions. Most maintenance engineers in building service systems, for example, usually do not indicate that the restoration interval of a PM is 2 years, they tend to estimate the restoration interval falls within an interval $(1,3)$ years instead. In this case, it can assume that the restoration interval is a random variable with a uniform probability distribution.

This paper considers the scenarios when the maintenance quality is a random variable. It assumes that both the adjustment factor $\theta$ and the restoration interval $\eta$ are random variables with certain probability distributions. Optimal PM policies for these two models are then obtained.

The paper is organized as follows. Section 2 introduces two novel PM models that consider failure rate PM models and age reduction PM models whose parameters for measuring the maintenance quality are random variables, and 
provides with algorithms for optimising PM policies. Further discussions on the quality of PM's are made in Section 3. Section 4 investigates two cases where the quality of PM's are assumed to be uniformly distributed and the failure time to be Weibull distributions. Finally, in the last section, concluding remarks are given.

\section{MODEL FORMULATIONS}

In this section, we investigate the failure rate PM model and the Canfield age reduction PM model, when the adjustment factor and the restoration interval are random variables. Model A assumes that the failure rate of a piece of equipment after a PM action become a product of a maintenance quality and the failure rate before the PM action. Model B assumes that the equipment's age become younger than before a PM action.

\subsection{Model A}

\section{Assumptions}

A. PM actions are performed at time $k T$ for $k=1, \ldots, N$. The equipment is replaced at time $N T$, where $T$ is the time interval between two adjacent PM actions and $N$ is the number of PM actions before a replacement.

B. When there is no PM or CM, the failure rate of the equipment, denoted by $h(t)$, is strictly increasing.

C. The equipment has the failure rate $h_{k}(t)=\theta^{k-1} h(t)$ after the $k$ th $\mathrm{PM}$, where $t \in(0, T)$, and $\theta$ is a random variable with a cumulative distribution function, denoted by $F(\theta)$ with $\theta \geq 1$. Assume that the $N$ th moment about 
the random variable $\theta$ exists.

D. A replacement can restore the equipment to be as good as new. The equipment undergoes minimal repair upon failures between two adjacent PM's. The failure rate remains unchanged by minimal repair.

E. The times for PM, minimal repair and replacement are negligible.

F. The costs on a minimal repair, a PM and a replacement are $c_{m}, c_{p}$ and $c_{r}$, respectively.

The long-run average cost rate is

$$
C_{A}(T, N)=\frac{1}{N T}\left\{c_{m} \sum_{k=1}^{N} \int_{0}^{T}\left(\int_{1}^{\infty} \theta d F(\theta)\right)^{k-1} h(t) d t+(N-1) c_{p}+c_{r}\right\}
$$

Denote $\gamma_{k}=\left(\int_{1}^{\infty} \theta d F(\theta)\right)^{k-1}$ and $r_{k}(t)=\gamma_{k} h(t)$. Eq. (1) can be re-written as

$$
C_{A}(T, N)=\frac{1}{N T}\left\{\left(c_{m} \sum_{k=1}^{N} \int_{0}^{T} r_{k}(t) d t\right)+(N-1) c_{p}+c_{r}\right\}
$$

To determine the optimal values of $N$ and $T$ that minimize $C_{A}(T, N)$ in Eq. (2), one can solve the following optimisation problem.

$$
C_{A}\left(T_{0}, N_{0}\right)=\min _{T, N} C_{A}(T, N)
$$

According to Nakagawa [5], there exists optimal $T_{0}$ and $N_{0}$ when Eq. (4) and (5) are satisfied.

$$
\begin{aligned}
& \int_{0}^{T}\left\{(N-1) r_{N}(t)-\sum_{k=1}^{N-1} r_{k}(t)\right\} d t<\frac{c_{r}-c_{p}}{c_{m}} \\
\leq & \int_{0}^{T}\left\{N r_{N+1}(t)-\sum_{k=1}^{N} r_{k}(t)\right\} d t
\end{aligned}
$$


and

$$
\sum_{k=1}^{N}\left\{\operatorname{Tr}_{k}(T)-\int_{0}^{T} r_{k}(t) d t\right\}=\frac{(N-1) c_{p}+c_{r}}{c_{m}}
$$

Table 1 shows an algorithm for searching the optimal $T_{0}$ and $N_{0}$ of Model A.

When the parameter $\theta$ is assumed to be a constant

\subsection{Model B}

Assume that Canfield's [6] age reduction PM model is utilized to maintain the equipment. Under Canfield's model, each PM reduces operational stress to that existing time units previous to the PM intervention, where the restoration interval is less than or equal to the PM intervention interval. Thus, each PM restores the failure rate of the equipment at time $t$ to the one at $t-\eta$, where $\eta \leq T$, while its shape remains unchanged. That is, the failure rate keeps monotonically increasing, although the rate of degradation is reduced after each PM. Canfield [6] derived the following failure rate:

$$
h_{k}(t)= \begin{cases}h(t) & \text { for } 0 \leq t \leq T \\ w_{k}(T, \eta)+h(t-k \eta) & \text { for } k T \leq t \leq(k+1) T\end{cases}
$$

where $0<\eta \leq T$, and $w_{k}(T, \eta)=\sum_{i=1}^{k}\{h(i T-(i-1) \eta)-h(i(T-\eta))\}$.

\section{Assumptions}

A. Assumptions A, B, D, E and F are the same as for Model A.

B. The equipment has the failure rate shown as Eq. (6), where $\eta$ is a random 
variable with cumulative distribution function $G(\eta)$ and $0<\eta \leq T$.

Based on the above assumption, we can obtain the following long-run average rate

$$
\begin{aligned}
C_{B}(T, N) & =\frac{1}{N T}\left\{c_{m} \int_{0}^{T}\left(\sum_{k=1}^{N-1} w_{k}(T, \eta) T+\sum_{k=0}^{N-1} \int_{k T}^{(k+1) T} h(t-k \eta) d t\right) d G(\eta)\right. \\
& \left.+(N-1) c_{p}+c_{r}\right\}
\end{aligned}
$$

For a fixed $N$, by solving the following equation, the time period $T_{0}$ that achieves the minimal long-run average cost rate can be obtained.

$$
C_{B}\left(T_{0}, N\right)=\min _{T} C_{B}(T, N)
$$

To determine the values of $N$ and $T$ that minimize $C_{B}(T, N)$ in Eq. (8), denote

$$
\begin{aligned}
L(T, N) & =N \int_{0}^{T}\left\{w_{N}(T, \eta) T+\int_{0}^{T} h(t+N T-N \eta) d t\right\} d G(\eta) \\
& -\int_{0}^{T}\left\{\sum_{k=1}^{N-1} w_{k}(T, \eta) T+\sum_{k=0}^{N-1} \int_{0}^{T} h(t+k T-k \eta) d t\right\} d G(\eta)
\end{aligned}
$$

Let $C_{B}(T, N+1) \geq C_{B}(T, N)$ and $C_{B}(T, N-1)>C_{B}(T, N)$, which implies

$$
L(T, N) \geq \frac{c_{r}-c_{p}}{c_{m}} \text { and } L(T, N-1)<\frac{c_{r}-c_{p}}{c_{m}}
$$

Let $\frac{\partial C_{B}(T, N)}{\partial T}=0$, then

$$
\begin{aligned}
& \frac{N(N-1)}{2} T^{2} h(T) g(T)+T g(0) \int_{0}^{N T} h(t) d t+N T g(T) \int_{0}^{T} h(t) d t \\
- & \int_{0}^{T}\left(\sum_{k=1}^{N-1} w_{k}(T, \eta) T+\sum_{k=0}^{N-1} \int_{0}^{T} h(t+k T-k \eta) d t\right) d G(\eta) \\
= & \frac{(N-1) c_{p}+c_{r}}{c_{m}}
\end{aligned}
$$


where $g(T)=\left.\frac{d G(t)}{d t}\right|_{t=T}$, and $g(0)=\left.\frac{d G(t)}{d t}\right|_{t=0}$. Therefore, there exists optimal $T_{0}$ and $N_{0}$ when Eq. (9) and (10) are satisfied.

Table 2 shows an algorithm for searching the optimal $T_{0}$ and $N_{0}$ of Model B.

\section{DISCUSSION}

In the failure rate PM model and the age reduction PM model, both the adjustment factor $\theta$ and the restoration interval $\eta$ are assumed to be constant. The above assumption that parameters $\theta$ and $\eta$ are random variables provides an alternative approach to investigating the two parameters. The restoration interval $\eta$ in the age reduction model can be estimated by either domain experts [7] or collected data [6]. As domain experts tend to offer a rough estimate of parameters, the above assumption may be more suitable for the situation when the domain experts are asked to estimate the parameters.

For the failure rate PM model, this paper only considers Canfield's age reduction model. Similarly, the parameter $b$ in the Malik's age reduction model can be regarded as a random variable and analysed. As above mentioned, the hybrid PM model $[13,14]$ is derived on the basis of the failure rate PM model and the age reduction PM model. The PM policies based on the hybrid PM model can be optimised if parameters $a_{k}$ and $b_{k}$ are random variables.

Further research can be considered in the case when the equipment's age may impact the adjustment factor or the restoration interval. Chan and Shaw[15], and $\mathrm{Gu}[16]$ considered the scenarios where the adjustment factor varies with the equipment's age; sequential PM modelling (see $[13,14,5]$ ) is a solution for such scenarios. However, each PM action in sequential PM modelling has a 
different quality, which requires a large number of data to estimate it. In practical use, it may be very hard to provide system designers or maintenance engineers with a huge number of data.

\section{NUMERICAL EXAMPLES}

Suppose that the failure time of a piece of equipment has a Weibull distribution, i.e. $h(t)=\beta t^{\beta-1}$, where $\beta=1.6$.

\subsection{Model A}

Let the adjustment factor $\theta$ in Model $\mathrm{A}$ has a uniform distribution as follows:

$$
F(\theta)=\left\{\begin{array}{l}
\frac{\theta-1}{u-1} \text { for } 1 \leq \theta \leq u \\
0 \quad \text { otherwise }
\end{array}\right.
$$

Then for $\gamma_{k}=\left(\frac{1+u}{2}\right)^{k-1}$ and $r_{k}(t)=\beta \gamma_{k} t^{\beta-1}$. The optimal solution $N_{0}$ and $T_{0}$ should satisfy the following inequality and equation:

$$
\left(N_{0}-1\right) T_{0}{ }^{\beta} \gamma_{N_{0}}-T_{0}{ }^{\beta} \sum_{k=1}^{N_{0}-1} \gamma_{k}<\frac{c_{r}-c_{p}}{c_{m}} \leq N_{0} T_{0}{ }^{\beta} \gamma_{N_{0}+1}-T_{0}{ }^{\beta} \sum_{k=1}^{N_{0}} \gamma_{k}
$$

and

$$
T_{0}=\left\{\frac{\left(N_{0}-1\right) c_{p}+c_{r}}{c_{m}(\beta-1) \sum_{k=1}^{N_{0}} \gamma_{k}}\right\}^{\frac{1}{\beta}}
$$

Then the minimal long-run average cost is

$$
C_{A}\left(T_{0}, N_{0}\right)=\frac{1}{N_{0} T_{0}}\left\{c_{m} T_{0}{ }^{\beta} \sum_{k=1}^{N_{0}} \gamma_{k}+\left(N_{0}-1\right) c_{p}+c_{r}\right\}
$$


Based on research in the office building services systems, Evans et al(see [17]) have identified the relationships among three costs incurred within the systems' whole lifecycle span: initial capital costs, maintenance and building operating costs, and business operating costs. They found that the operation and maintenance of the building services systems costs can be five times the capital costs; while the business operating costs will be two hundred times the capital costs over the life of the building. In other words, business losses are forty times as large as maintenance costs, namely, corrective maintenance costs (that includes business losses) is forty times as large as preventive maintenance costs.

We therefore assume that $c_{p}=1, c_{m}=40$ and $c_{r}=1000$. When $u$ in the cumulative distribution function Eq. (11) changes from 1.1 to 2.0, one can obtain the following results shown in Table 3 and Figure 1 based on the above discussion. In order to show the data in a figure, the long-run average cost is divided by 10 in Figure 1.

From Figure 1, if $u$ increases, the replacement times $N_{0}$ decreases, and both the time intervals $T_{0}$ and $C_{A}\left(T_{0}, N_{0}\right)$ increase.

\subsection{Model B}

Let the restoration interval $\eta$ in Model B has a uniform distribution as follows:

$$
G(\eta)= \begin{cases}\eta / T \text { for } 0 \leq \eta \leq T \\ 0 \quad \text { otherwise }\end{cases}
$$


The distribution indicates that a PM action can bring the equipment to the condition between before this PM and after the last PM. Denote

$$
\begin{aligned}
D_{N_{0}} & =\sum_{k=2}^{N_{0}-1} \sum_{i=2}^{k} \frac{i^{\beta}-1}{i-1}-\sum_{k=1}^{N_{0}-1}\left(\sum_{i=1}^{k} i^{\beta-1}-\frac{(k+1)^{\beta+1}-k^{\beta+1}-1}{k(\beta+1)}\right) \\
& +\left(N_{0}-1\right) \beta+1
\end{aligned}
$$

Then, the optimal solution $N_{0}$ should satisfy the following inequality:

$$
\left(N_{0}-1\right) D_{N_{0}}-N_{0} D_{N_{0}-1} \leq \frac{c_{r}-c_{p}}{c_{m} T_{0}^{\beta}}<N_{0} D_{N_{0}+1}-\left(N_{0}+1\right) D_{N_{0}}
$$

And $T_{0}$ can be obtained from the following equation:

$$
T_{0}=\left\{\frac{\left(N_{0}-1\right) c_{p}+c_{r}}{c_{m}(\beta-1) D_{N_{0}}}\right\}^{1 / \beta}
$$

The long-run average cost is

$$
C_{B}\left(T_{0}, N_{0}\right)=\frac{c_{m} T_{0}^{\beta} D_{N_{0}}+\left(N_{0}-1\right) c_{p}+c_{r}}{N_{0} T_{0}}
$$

We assume that $c_{p}=1$ and $c_{m}=40$. When $c_{r}$ changes from 500 to 5000 , we have the following results shown in Table 4 and Figure 2 based on the above discussion. In order to show the data in a figure, the long-run average cost is divided by 10 in Figure 2 .

Model A and model B are developed based on the assumption that the parameters $\theta$ and $\eta$ are random variable. If the parameters are set to the expected values of the random variables, the number of the PM actions before a replacement and the time interval between two adjacent PM actions can be optimised. For example, set a fixed parameter $\eta=\frac{T}{2}$ for model B, then the optimal values of $N_{0}$ and $T_{0}$ are shown in Table 5 . Compare Table 4 and Table 5, one can find that the cost in Table 4 is lower than in Table 5, which 
indicates the effect on the results of choosing the uniform distributions for the parameter $\eta$ of model B is better than that of choosing a fixed value. Figure 3 shows the change of the long-run average cost divided by 10 when $\eta=\frac{T}{2}$.

\section{CONCLUSIONS}

In both the reliability literature and practical use, the quality of preventive maintenance $(\mathrm{PM})$ is an interesting topic because it is vitally important during optimizing maintenance policies and lifecycle costing.

Prior research assumes that the quality of PM is a fixed constant, which is usually not true in many scenarios. This paper investigated the optimization problem of PM policies for the situations where the quality of PM is a random variable with a certain probability distribution, which would be more practical than the situation when the quality of maintenance is assumed to be a fixed constant. The optimal maintenance policies for failure rate PM models and age reduction PM models were obtained in the paper.

When the life distribution of a piece of equipment is a Weibull distribution and the quality of PM distributes uniformly, explicit expressions of the optimal time interval of PM can be obtained. The numerical example shows how the long-run average cost changes with the quality of PM; it also shows that the effect on the long-run average cost of choosing the uniform distributions for the restoration interval of the age reduction model is lower than that of choosing a fixed value.

\section{ACKNOWLEDGMENTS}


The author would like to thank two anonymous reviewers for their comments and suggestions, which have resulted in a number of improvements in the paper. The authors would like to thank EPSRC as part of the Innovative Construction Research Centres (ICRC) initiative for their financial support and our industrial partners (EC Harris, Dytecna Limited, EMCOR Rail, INBIS and Quorum Logistics Support).

\section{References}

[1] Pham H, Wang H. Imperfect maintenance. European Journal of Operational Research 1996; 94:425-438.

[2] Dekker R. Applications of maintenance optimization models: A review and analysis. Reliability Engineering and System Safety 1996; 51:229-240

[3] Scarf PA. On the application of mathematical models in maintenance. European Journal of Operational Research 1997; 99:493-506

[4] Lie $\mathrm{CH}$, Chun YH. An algorithm for preventive maintenance policy. IEEE Transactions on Reliability 1986; R-35:71-75.

[5] Nakagawa T. Periodic and sequential preventive maintenance policies. Journal of Applied Probability 1986; 23:536-542.

[6] Canfield RV. Cost optimization of periodic preventive maintenance. IEEE Transactions on Reliability 1986; 35:78-81.

[7] Malik MAK. Reliable preventive maintenance scheduling. AIIE Transactions $1979 ; 11: 221-228$.

[8] Park DH, Jung GM, Yum JK. Cost minimization for periodic maintenance policy of a system subject to slow degradation. Reliability Engineering and System Safety 2000; 68:105-112. 
[9] Jung GM, Park DH. Optimal maintenance policies during the post-warranty period. Reliability Engineering and System Safety 2003; 82:173-185.

[10] Shin I, Lim TJ, Lie CH. Estimating parameters of intensity function and maintenance effect for repairable unit. Reliability Engineering and System Safety $1996 ; 54: 1-10$.

[11] Shin I, Lim TJ, Lie CH. Estimating parameters of intensity function and maintenance effect for repairable unit. Reliability Engineering and System Safety 1996; 54:1-10.

[12] Jayabalan V, Chaudhuri D. Optimal maintenancereplacement policy under imperfect maintenance. Reliability Engineering and System Safety 1992; 36:165169.

[13] Lin D, Zuo MJ, Yam RCM. General sequential imperfect preventive maintenance models. International Journal of Reliability Quality and Safety Engineering 2000; 7:253-266.

[14] Lin D, Zuo MJ, Yam RCM. Sequential imperfect preventive maintenance models with two categories of failure Modes. Naval Research Logistics 2001; 48:172-182.

[15] Chan J, Shaw L. Modeling repairable systems with failure rates that depend on age and maintenance. IEEE Transactions on Reliability 1993; R-42:566-571.

[16] Gu HY. Technical Note: Studies on optimum preventive maintenance policies for general repair reliability. Reliability Engineering and System Safety 1993; 41:197-201.

[17] Evans R, Haryott H, Haste N, Jones A. The long term costs of owning and using buildings. London: The Royal Academy of Engineering Publishing, 1998. 
Fig. 1. Changes of the optimal solution $\boldsymbol{N}_{0}, T_{0}$ and $C_{A}\left(T_{0}, N_{0}\right)$ with the change of $u$

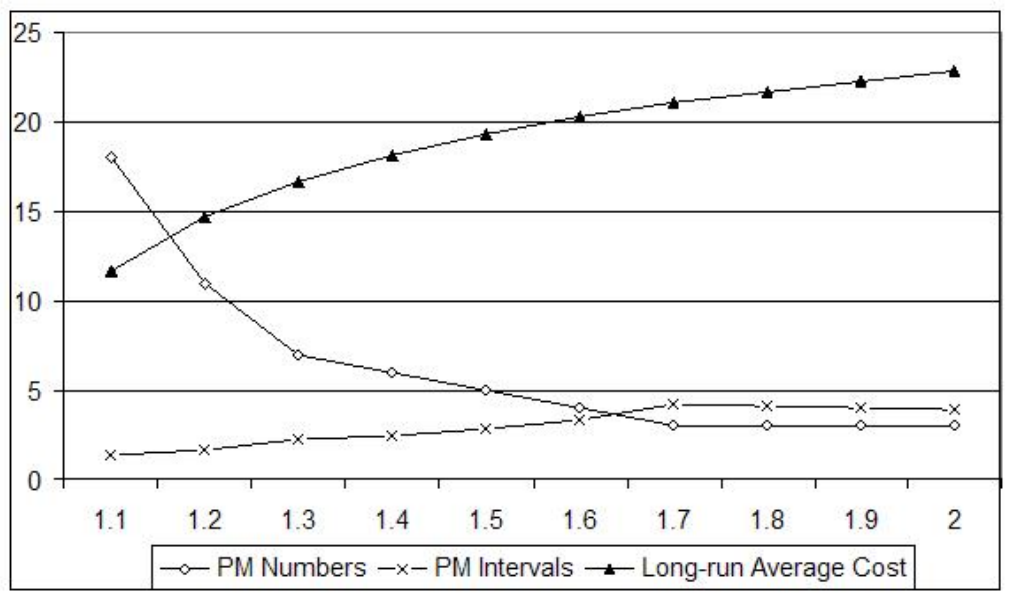

Fig. 2. Changes of the optimal solution $N_{0}, T_{0}$ and $C_{A}\left(T_{0}, N_{0}\right)$ with the change of $c_{r}$ when eta is a random variable with a uniform distribtuion as Eq. (15)

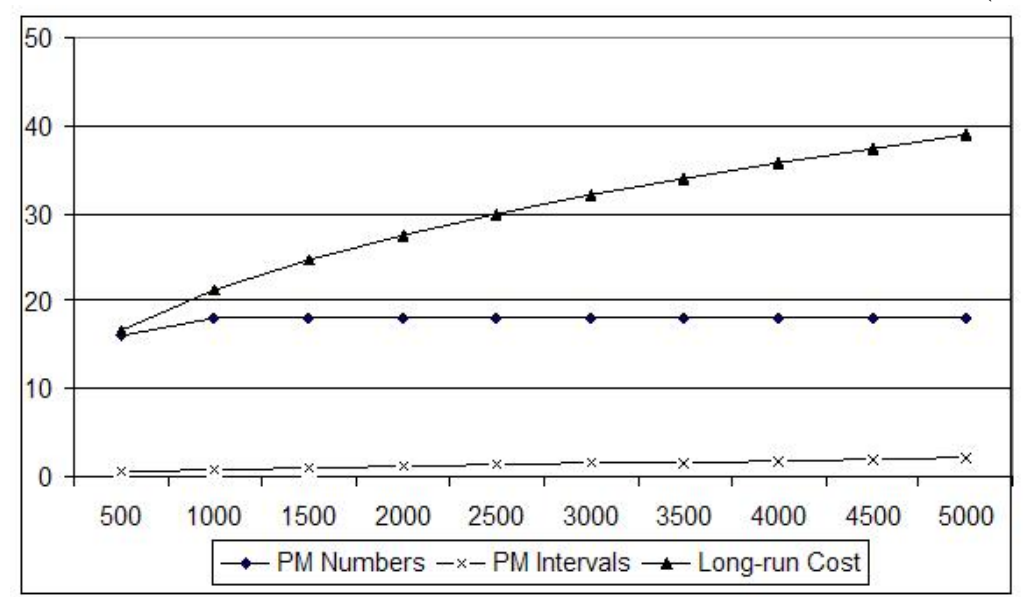


Fig. 3. Changes of the optimal solution $\boldsymbol{N}_{0}, T_{0}$ and $C_{A}\left(T_{0}, N_{0}\right)$ the change of $c_{r}$ when $\eta=\frac{T}{2}$

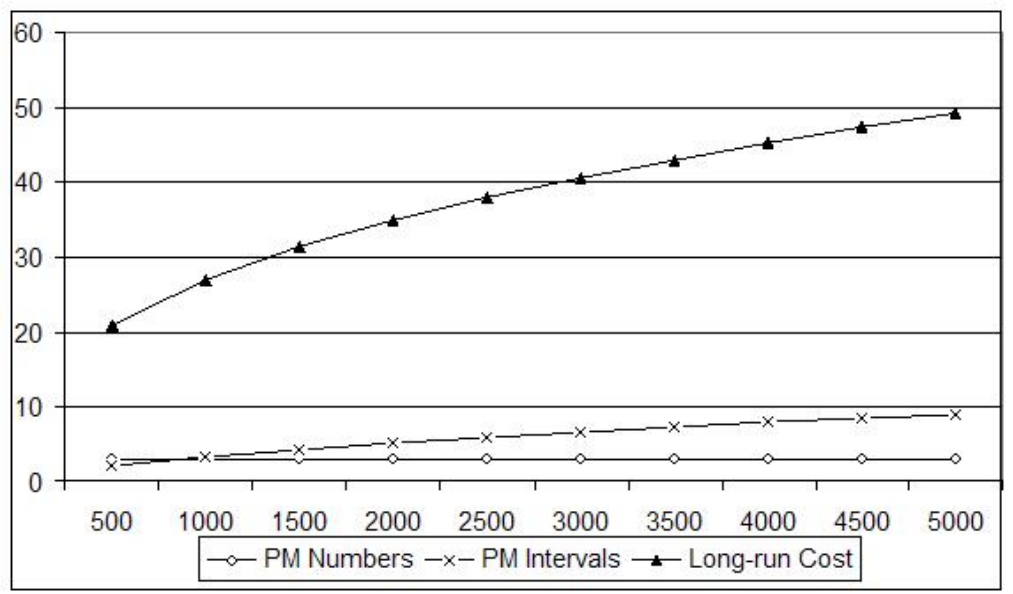


Table 1

Searching the optimal $T_{0}$ and $N_{0}$ for Model A

\section{Inputs}

$h(t)$ : the failure rate $c_{m}$ : the cost of minimal repair;

$c_{p}: \quad$ the cost of PM; $\quad c_{r}: \quad$ the cost of replacement;

$F(\theta)$ : the cumulative distribution function of $\theta$

\section{Outputs}

$N_{0}$ : the cumulative distribution function of $\theta$

$T_{0}$ : the optimal time interval for periodic PM;

1: for $N=\{1,2, \ldots, \infty\}$ do

2: $\quad$ obtain $T$ by solving Eq. (5);

3: if inequality (4) is satisfied, then

4: $\quad$ calculate $C_{A}(T, N)$ from Eq. (2);

5: $\quad T_{0} \Leftarrow T ; N_{0} \Leftarrow N ;$

6: break;

7: $\quad$ end

8: end 
Table 2

Searching the optimal $T_{0}$ and $N_{0}$ for Model B

For Inputs, replace $F(\theta)$ with $G(\eta)$, and $\theta$ with $\eta$. Outputs are the same

as Algorithm 1. The calculating steps are the same as Algorithm 1 after the follow

interchanges have been made: Eq. (4) with Eq. (9); Eq. (5)

with Eq. (10); Eq. (2) with Eq. (7), respectively.

Table 3

Changes of the optimal solution $\boldsymbol{N}_{0}, T_{0}$ and $C_{A}\left(T_{0}, N_{0}\right)$ with the change of $u$

\begin{tabular}{ccccccccccc}
\hline$u$ & 1.1 & 1.2 & 1.3 & 1.4 & 1.5 & 1.6 & 1.7 & 1.8 & 1.9 & 2.0 \\
\hline$N_{0}$ & 18 & 11 & 7 & 6 & 5 & 4 & 3 & 3 & 3 & 3 \\
$T_{0}$ & 1.4 & 1.7 & 2.3 & 2.5 & 2.8 & 3.3 & 4.2 & 4.1 & 4 & 3.9 \\
\hline$C_{A}\left(T_{0}, N_{0}\right)$ & 116.8 & 146.6 & 166.7 & 181.7 & 193.5 & 202.6 & 211.1 & 217.1 & 223.0 & 228.9 \\
\hline
\end{tabular}

Table 4

Changes of the optimal solution $\boldsymbol{N}_{0}, T_{0}$ and $C_{B}\left(T_{0}, N_{0}\right)$ with the change of $c_{r}$ when eta is a random variable with a uniform distribtuion as Eq. (15)

\begin{tabular}{ccccccccccc}
\hline$c_{r}$ & 500 & 1000 & 1500 & 2000 & 2500 & 3000 & 3500 & 4000 & 4500 & 5000 \\
\hline$N_{0}$ & 3 & 3 & 3 & 3 & 3 & 3 & 3 & 3 & 3 & 3 \\
\hline$T_{0}$ & 2.2 & 3.3 & 4.2 & 5.1 & 5.9 & 6.6 & 7.2 & 7.9 & 8.5 & 9.0 \\
\hline$C_{B}\left(T_{0}, N_{0}\right)$ & 207.6 & 268.9 & 313.0 & 348.6 & 378.9 & 405.8 & 429.9 & 451.9 & 472.4 & 491.4 \\
\hline
\end{tabular}


Table 5

Changes of the optimal solution $N_{0}, T_{0}$ and $C_{B}\left(T_{0}, N_{0}\right)$ with the change of $c_{r}$ when $\eta=\frac{T}{2}$

\begin{tabular}{ccccccccccc}
$c_{r}$ & 500 & 1000 & 1500 & 2000 & 2500 & 3000 & 3500 & 4000 & 4500 & 5000 \\
\hline$N_{0}$ & 16 & 18 & 18 & 18 & 18 & 18 & 18 & 18 & 18 & 18 \\
\hline$T_{0}$ & 0.6 & 0.8 & 1.0 & 1.2 & 1.4 & 1.6 & 1.7 & 1.9 & 2.0 & 2.2 \\
\hline$C_{B}\left(T_{0}, N_{0}\right)$ & 167.3 & 213.6 & 247.8 & 275.8 & 299.9 & 321.6 & 339.7 & 357.8 & 373.3 & 389.1 \\
\hline
\end{tabular}


Table 5

Changes of the optimal solution $N_{0}, T_{0}$ and $C_{B}\left(T_{0}, N_{0}\right)$ with the change of $c_{r}$ when $\eta=\frac{T}{2}$

\begin{tabular}{ccccccccccc}
$c_{r}$ & 500 & 1000 & 1500 & 2000 & 2500 & 3000 & 3500 & 4000 & 4500 & 5000 \\
\hline$N_{0}$ & 16 & 18 & 18 & 18 & 18 & 18 & 18 & 18 & 18 & 18 \\
\hline$T_{0}$ & 0.6 & 0.8 & 1.0 & 1.2 & 1.4 & 1.6 & 1.7 & 1.9 & 2.0 & 2.2 \\
\hline$C_{B}\left(T_{0}, N_{0}\right)$ & 167.3 & 213.6 & 247.8 & 275.8 & 299.9 & 321.6 & 339.7 & 357.8 & 373.3 & 389.1 \\
\hline
\end{tabular}

